\title{
An Alternative Triage Strategy Based on Preoperative MRI for Avoiding Trimodality Therapy in Stage IB Cervical Cancer
}

\section{Jung-Yun Lee, MD \\ Jina Youm, MD² \\ Jae Weon Kim, MD, PhD² \\ Kidong Kim, MD, PhD ${ }^{3}$ \\ Hak Jae Kim, MD, PhD ${ }^{4}$ \\ Jeong Yeon Cho, MD, PhD \\ Min A Kim, MD, PhD ${ }^{6}$ \\ Noh Hyun Park, MD, PhD² \\ Yong-Sang Song, MD, PhD²}

\section{${ }^{1}$ Department of Obstetrics and Gynecology,} Institute of Women's Life Medical Science, Yonsei University College of Medicine, Seoul, ${ }^{2}$ Department of Obstetrics and Gynecology, Seoul National University

College of Medicine, Seoul,

${ }^{3}$ Department of Obstetrics and Gynecology, Seoul National University Bundang Hospital, Seongnam, Departments of

${ }^{4}$ Radiation Oncology, ${ }^{5}$ Radiology, and

${ }^{6}$ Pathology, Seoul National University

College of Medicine, Seoul, Korea

\begin{abstract}
Purpose
Adjuvant chemoradiation following primary surgery is frequently indicated in patients with stage IB cervical cancer. The aim of this study is to evaluate the role of a magnetic resonance imaging (MRI)-based strategy in avoiding trimodality therapy.
\end{abstract}

\begin{abstract}
Materials and Methods
We retrospectively reviewed all patients with stage IB cervical cancer treated initially with primary surgery at Seoul National University Hospital. We suggest an alternative triage strategy in which the primary treatment modality is determined based on preoperative MRI findings. Using this strategy, primary surgery is only indicated when there is no evidence of parametrial involvement (PMI) and lymph node metastasis (LNM) in the MRI results; when there is evidence of either or both of these factors, primary chemoradiation is selected. Assuming that this strategy is applied to our cohort, we evaluate how the rate of trimodality therapy is affected.
\end{abstract}

\section{Results}

Of the 254 patients in our sample, 77 (30.3\%) had at least one category 1 risk factor (PMI, LNM, positive resection margin) upon pathologic examination. If the MRI-based strategy had been applied to our cohort, 168 patients would have undergone primary surgery and 86 would have undergone primary chemoradiation. Only 25 patients (9.8\%) would have required trimodality therapy based on an indication of at least one category 1 pathologic risk factor following radical hysterectomy.

\section{Conclusion}

The inclusion of MRI in the decision-making process for primary treatment modality could have reduced the number of patients requiring trimodality therapy based on the indication of a category 1 risk factor from $30.3 \%$ to $9.8 \%$ in our cohort.
Correspondence: Jae Weon Kim, MD, PhD Department of Obstetrics and Gynecology, Seoul National University College of Medicine, 101 Daehak-ro, Jongno-gu, Seoul 03080, Korea Tel: 82-2-2072-3511

Fax: 82-2-762-3599

E-mail: kjwksh@snu.ac.kr

Received December 29, 2014

Accepted February 24, 2015

Published Online March 20, 2015

\section{Key words}

Uterine cervical neoplasms, Chemoradiotherapy,

Radical hysterectomy, Trimodality therapy,

Magnetic resonance imaging, Triage

\section{Introduction}

Cervical cancer is the third most common female malignancy worldwide and the most common female genital tract malignancy in Korean women [1,2]. The primary means of treating early-stage cervical cancer is either surgery or radi- ation therapy. Surgery is preferred for lower-stage disease with smaller lesions, such as stage IA, IB1, and selected IIA1, as ovarian and vaginal function can be preserved $[3,4]$. For patients with stage IB2 or IIA2 disease, primary chemoradiation is preferable to radical hysterectomy followed by adjuvant chemoradiation - known as trimodality therapy—as the latter has a higher morbidity rate [5-7]. 
To avoid morbidity resulting from trimodality therapy, primary surgery should be limited to patients with the lowest possible risk of needing adjuvant therapy. The International Federation of Gynecology and Obstetrics (FIGO) staging system is based on clinical staging, including visual inspection, colposcopy, cervical biopsy, and manual pelvic examination, and limits the imaging to chest radiography, intravenous pyelography, and barium enema [8]. However, a considerable proportion of patients with clinical stage IB cervical cancer already have pathologic risk factors, such as parametrial involvement (PMI) or lymph node metastasis (LNM), before surgery. In stage IB2 and IIA2 cervical cancer in particular, more than half of patients undergo postoperative adjuvant therapy following primary radical surgery $[9,10]$. Although it is not accepted as part of the formal staging procedure, the use of advanced imaging techniques, such as magnetic resonance imaging (MRI), computed tomography (CT), and positron emission tomography (PET)-CT, has been suggested as a means of guiding treatment options and design for cervical cancer patients [11-13].

We developed a decision-analytic model to determine the role of the triage strategy based on preoperative MRI findings in a cohort of patients with clinical stage IB cervical cancer. The aim of this study is to evaluate how the rate of trimodality therapy is affected when this alternative triage strategy is used.

\section{Materials and Methods}

\section{Patients}

After receiving approval from the Institutional Review Board, all patients treated surgically for FIGO stage IB cervical cancer between 2003 and 2011 at Seoul National University Hospital were reviewed retrospectively. The use of MRI in diagnosis and follow-up has been widespread at this institution since 2003. Patients eligible for inclusion were those: (1) with stage IB cervical cancer and clinically visible lesions; (2) who had undergone primary radical hysterectomy followed by tailored adjuvant therapy; and (3) who had a preoperative MRI up to 4 weeks before surgery. Patients who had undergone radiation therapy or chemotherapy before surgery or conization before MRI were excluded. Consequently, 254 patients were eligible for analysis. We included 190 cases from a previous study of ours published in 2014 [14].

\section{MRI and image analysis}

The MRI data were reviewed by a radiologist (J.Y.C.), who was blind to surgical outcomes. The same rating scale for PMI suggested in a previous study was used $[14,15]$. A lesion observed in MRI findings was considered positive when a pelvic or para-aortic lymph node (LN) displayed a short-axis dimension $\geq 1 \mathrm{~cm}$. Any LN measuring $1 \mathrm{~cm}$ or marginally less, giving an overall equivocal impression, was considered negative.

Patients were categorized according to two groups based on MRI-based parameters: a low-risk group (no evidence of PMI and LNM in MRI) and a high-risk group (findings suggesting PMI and/or LNM in MRI).

\section{Decision-analysis model}

We developed a decision-analysis model to compare the rate of trimodality therapy for stage IB cervical cancer patients when two different strategies were employed (1) a primary surgery strategy, in which radical surgery and pelvic lymphadenectomy is recommended to all patients; and (2) an MRI-based strategy, in which primary treatment modality is recommended according to risk criteria based on preoperative MRI results. Under the latter, patients classified as low risk based on MRI findings were assumed to undergo primary radical surgery followed by tailored adjuvant therapy, and those classified as high risk were assumed to undergo primary chemoradiation. In addition, we analyzed the number of MRIs that would need to be performed in order to spare one patient from undergoing trimodality therapy.

\section{Adjuvant therapy following primary surgery}

All patients were assumed to undergo adjuvant treatment in line with their pathologic risk factors. Patients with at least one category 1 risk factor (positive resection margin, LNM, or PMI) received adjuvant chemoradiation. Patients with two or more category 2 risk factors (positive lymphovascular space invasion, deep stromal invasion, or large tumor size) received adjuvant chemoradiation. Guideline adherence to adjuvant treatment was assumed to be $100 \%$.

\section{Results}

The characteristics of the study population are summarized in Table 1 . The median age was 49 years (range, 25 to 78 years) and 64 patients with stage IB2 were included. Squa- 
Table 1. Baseline characteristics

$\begin{array}{lc}\text { Variable } & \text { No. }(\%) \\ \text { Median age (range, yr) } & 49(25-78) \\ \text { Clinical stage } & \\ \text { IB1 } & 64(74.8) \\ \text { IB2 } & \\ \text { Histology } & 181(71.3) \\ \text { Squamous cell carcinoma } & 56(22.0) \\ \text { Adenocarcinoma } & 13(5.1) \\ \text { Adenosquamous carcinoma } & 4(1.6) \\ \text { Others } & \\ \text { MRI-based parameters } & \\ \text { Parametrial involvement } & 208(81.9) \\ \text { No } & 46(18.1) \\ \text { Yes } & 195(76.8) \\ \text { Lymph node metastasis } & 59(23.2) \\ \text { No } & \\ \text { Yes }\end{array}$

MRI, magnetic resonance imaging.

mous cell carcinoma was the most prevalent histologic subtype $(71.3 \%)$, followed by adenocarcinoma $(22.0 \%)$ and adenosquamous carcinoma (5.1\%). Of all patients, 18.1\% (46 patients) had MRI findings indicating PMI and 23.2\% (59 patients) had MRI findings suggesting LNM.

A decision tree comparing the two strategies for newly diagnosed, stage IB cervical cancer patients is shown in Fig. 1. Under the primary surgery strategy, all patients would have undergone radical hysterectomy and pelvic lymphadenectomy (in reality, all 254 patients in our cohort underwent primary surgery regardless of the MRI findings). Of the total study population, 77 patients $(30.3 \%$ ) would have undergone adjuvant chemoradiation based on at least one category 1 pathologic risk factor. Conversely, under the alternative, MRI-based strategy, 168 patients $(66.1 \%)$ classified as low risk based on their MRI findings would have undergone primary radical surgery followed by tailored adjuvant therapy, and 86 patients $(33.9 \%)$ classified as high risk would have undergone primary chemoradiation. Only 25 patients would have required adjuvant chemoradiation based on at least one category 1 pathologic risk factor following surgery, while of the 143 low-risk patients who did not have category 1 pathologic risk factors, $33(23.1 \%)$ would have required adjuvant chemoradiation based on two or more category 2 risk factors.

Comparison of the rates of trimodality therapy when the two strategies are applied to our cohort is shown in Table 2. For stage IB disease, considering MRI findings when deciding between primary surgery or primary chemoradiation could reduce the rate of trimodality therapy based on at least one category 1 risk factor from $30.3 \%$ to $9.8 \%$ and increase the proportion of patients not undergoing trimodality therapy from $50.4 \%$ to $77.2 \%$. For patients with stage IB2 disease, this effect was particularly prominent. Using MRI-based triage in these cases could reduce the proportion of patients requiring trimodality therapy based on category 1 risk factors from $54.7 \%$ to $10.9 \%$ and increase the proportion of patients not undergoing trimodality therapy from $14.1 \%$ to $70.3 \%$. From this, we can deduce that 4.9 MRIs would have had to be performed in order to spare one patient from trimodality therapy in stage IB cervical cancer.

\section{Discussion}

For early-stage cervical cancer, initial treatment options include primary surgery followed by tailored adjuvant therapy and primary chemoradiation. The current National Comprehensive Cancer Network (NCCN) guidelines recommend basing the primary treatment option on tumor size for stage IB cervical cancer patients [16]. Surgery is the preferred option for patients with stage IB1 disease, whereas primary chemoradiation is the most appropriate option for those with stage IB2 disease. However, in practice, primary surgery followed by tailored adjuvant therapy is the preferred and most frequently used treatment modality for stage IB2 patients [17-19]. Therefore, a considerable proportion of patients undergo adjuvant therapy following surgery and risk high morbidity rates as a result of trimodality therapy $[10,20,21]$. If the MRI-based strategy had been applied to our cohort, 168 patients would have undergone primary surgery and 86 would have undergone primary chemoradiation. Only 25 patients $(9.8 \%)$ would have required trimodality therapy based on the indication of at least one category 1 pathologic risk factor following radical hysterectomy.

Only a single randomized controlled trial (RCT) has compared the outcomes of primary surgery and primary radiotherapy [7]. This study did not find greater survival rates for either treatment modality, however an increase in toxicity was observed following the combined use of radical hysterectomy and adjuvant radiation. To date, no RCT comparing the outcomes of primary surgery and primary chemoradiation has been conducted, however small retrospective case-control studies reported no significant difference in outcomes between the two treatment options $[10,21]$. Although several investigators have shown that primary surgery followed by tailored adjuvant therapy improves outcomes in the era of chemoradiation, it is generally accepted that outcomes of either modality in treating early-stage 


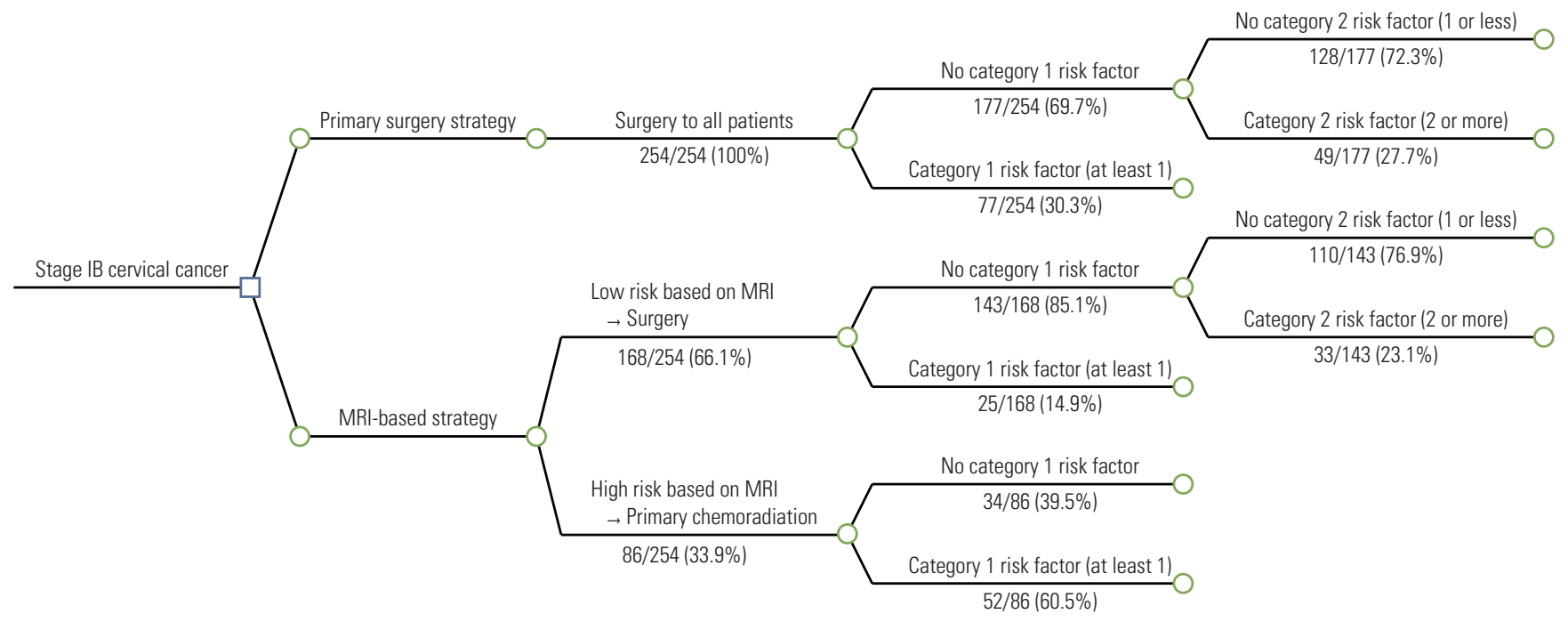

Fig. 1. A decision tree comparing the two strategies (primary surgery strategy and magnetic resonance imaging [MRI]based strategy) for stage IB cervical cancer. Category 1 risk factors: positive resection margin, lymph node metastasis, parametrial involvement; category 2 risk factors: positive lymphovascular space invasion, deep stromal invasion, large tumor size.

disease are comparable [9,22]. The Korean Gynecologic Oncology Group (KGOG) 1029 trial is currently recruiting patients with bulky early-stage cervical cancer in order to compare the outcomes of primary surgery and primary chemoradiation.

Some studies insist that primary surgery continues to play a significant role in treatment of patients with stage IB cervical cancer and even bulky stage IB2 disease. Park et al. [9] reported that $29.3 \%$ of patients with tumors $>4 \mathrm{~cm}$ were cured by surgery alone, and such patients have the best survival outcomes and lowest morbidity rates by avoiding radiation therapy. Therefore, in the preoperative stage we should identify the subset of patients with stage IB2 disease who may benefit from surgery alone.

In the absence of conclusive RCTs that determine the best treatment option for patients with bulky cervical cancer, restricting primary surgery to patients who can be treated successfully with surgery alone may be the most reasonable option. With this in mind, we should consider the best triage strategy for determining when primary surgery is an option and when primary chemoradiation is preferable, thus avoiding trimodality therapy for patients with stage IB cervical cancer. Previous studies have suggested that trimodality therapy is associated with high morbidity rates compared to surgery alone and primary chemoradiation as well as serious toxicity frequencies $>20 \%[7,10,23]$. Considering the complication rates of trimodality therapy, we suggest that primary surgery should be the initial treatment option for patients who are less likely to have adjuvant therapy, while primary chemoradiation is preferable for those who are likely to have to undergo adjuvant therapy following surgery. Preoperative prediction models are required for accurate allocation of primary treatment options for patients with stage IB disease.

Under an alternative, MRI-based strategy, the rate of trimodality therapy based on category 1 and 2 risk factors would be reduced, especially for stage IB2 disease. However, the proportion of patients who undergo surgery alone under an MRI-based strategy $(\mathrm{n}=110,43.3 \%)$ is less than under a primary surgery strategy $(\mathrm{n}=128,50.4 \%)$. This is the main weakness of the MRI-based strategy: $7.1 \%$ of patients would not benefit from a purely surgical treatment modality and would instead undergo primary chemoradiation. MRI-based strategy has inherent weakness from inaccurate prediction for PMI and LNM. Regarding detection of metastatic lymph nodes, the recent meta-analysis shows that PET/CT has higher accuracy than MRI [24]. Our model based on preoperative MRI findings may need to be updated with addition of PET/CT parameters.

This study has some limitations. First, our model has some limitations. In this model, the benefit of primary surgery followed by tailored adjuvant therapy may be underestimated. For the simplicity of the model, we did not consider unstaged para-aortic LNM and salvage therapy after primary chemoradiation. Major drawbacks of primary concurrent chemoradiation are unstaged para-aortic LNM based on preoperative imaging study and salvage therapy from 
Table 2. Rate of multimodality therapy when the two strategies are applied to stage IB cervical cancer patients

\begin{tabular}{|c|c|c|}
\hline Patient & No. $(\%)$ & Change $(\%)$ \\
\hline \multicolumn{3}{|l|}{ Stage IB (n=254) } \\
\hline \multicolumn{3}{|l|}{ Primary surgery strategy } \\
\hline Undergoing primary surgery & $254(100)$ & - \\
\hline Requiring trimodality therapy due to at least one category 1 risk factor & $77(30.3)$ & - \\
\hline Requiring trimodality therapy due to two or more category 2 risk factor & $49(19.3)$ & - \\
\hline Not undergoing trimodality therapy & $128(50.4)$ & - \\
\hline \multicolumn{3}{|l|}{ MRI-based strategy } \\
\hline Undergoing primary surgery & $168(66.1)$ & -33.9 \\
\hline Requiring trimodality therapy due to at least one category 1 risk factor & $25(9.8)$ & -20.5 \\
\hline Requiring trimodality therapy due to two or more category 2 risk factor & $33(13.0)$ & -6.3 \\
\hline Not undergoing trimodality therapy & $196(77.2)$ & 26.8 \\
\hline \multicolumn{3}{|l|}{ Stage IB1 $(n=190)$} \\
\hline \multicolumn{3}{|l|}{ Primary surgery strategy } \\
\hline Undergoing primary surgery & $190(100)$ & - \\
\hline Requiring trimodality therapy due to at least one category 1 risk factor & $42(22.1)$ & - \\
\hline Requiring trimodality therapy due to two or more category 2 risk factor & $29(15.3)$ & - \\
\hline Not undergoing trimodality therapy & $119(62.6)$ & - \\
\hline \multicolumn{3}{|l|}{ MRI-based strategy } \\
\hline Undergoing primary surgery & $141(74.2)$ & -25.8 \\
\hline Requiring trimodality therapy due to at least one category 1 risk factor & $18(9.5)$ & -12.6 \\
\hline Requiring trimodality therapy due to two or more category 2 risk factor & $21(11.1)$ & -4.2 \\
\hline Not undergoing trimodality therapy & $151(79.5)$ & 16.9 \\
\hline \multicolumn{3}{|l|}{ Stage IB2 $(n=64)$} \\
\hline \multicolumn{3}{|l|}{ Primary surgery strategy } \\
\hline Undergoing primary surgery & $64(100)$ & - \\
\hline Requiring trimodality therapy due to at least one category 1 risk factor & $35(54.7)$ & - \\
\hline Requiring trimodality therapy due to two or more category 2 risk factor & $20(31.3)$ & - \\
\hline Not undergoing trimodality therapy & $9(14.1)$ & - \\
\hline \multicolumn{3}{|l|}{ MRI-based strategy } \\
\hline Undergoing primary surgery & $27(42.2)$ & -57.8 \\
\hline Requiring trimodality therapy due to at least one category 1 risk factor & $7(10.9)$ & -43.8 \\
\hline Requiring trimodality therapy due to two or more category 2 risk factor & $12(18.8)$ & -12.5 \\
\hline Not undergoing trimodality therapy & $45(70.3)$ & 56.2 \\
\hline
\end{tabular}

Category 1 risk factors: positive resection margin, lymph node metastasis, parametrial involvement; category 2 risk factors: positive lymphovascular space invasion, deep stromal invasion, large tumor size. MRI, magnetic resonance imaging.

residual disease after completion of treatment. Second, the results were not validated in an independent set. The proportion of pathologic risk factors in stage IB disease varies according to the patient dataset and therefore affects the proportion of patients who require adjuvant therapy following surgery. Third, although predicting PMI and LNM using MRI gives accurate preoperative information, there is the possibility of inter-observer variation. To minimize this, all images were re-reviewed. Fourth, the current guidelines do not recommend using MRI as a universal preoperative means of evaluation for cervical cancer patients. Finally, despite reduced long-term gastrointestinal toxicity, especially in the postoperative adjuvant setting, radiation delivery techniques such as intensity-modulated radiation therapy were not considered.

Despite its limitations, the alternative triage strategy proposed in this paper has some key advantages. Marnitz et al. [25] suggest that laparoscopic staging is the best means for selection of patients for either primary chemoradiation or primary surgery and avoiding trimodality therapy. Radical hysterectomy was performed only in patients without evidence of LNM in the frozen section during laparoscopic 
staging. The authors show that the rate of trimodality therapy based on pathologic risk factors was $10 \%$ and suggest that this percentage could be used as a benchmark for the quality of interdisciplinary treatment for patients diagnosed with cervical cancer. By employing the MRI-based strategy, we can anticipate a low rate of trimodality therapy following primary surgery without performing laparoscopic staging.

\section{Conclusion}

In addition to maintaining the proportion of patients who could be treated using surgery alone, reducing morbidity arising from trimodality therapy should be a goal. Preoper- ative MRI provides useful information for determining the primary treatment modality for stage IB cervical cancer patients. Including MRI in the decision-making process may reduce morbidity arising from trimodality therapy in patients with early-stage cervical cancer. Further studies are needed in order to validate our results and a cost-effectiveness analysis should be performed, including the cost of MRI and complications arising from trimodality therapy.

\section{Conflicts of Interest}

Conflict of interest relevant to this article was not reported.

\section{References}

1. Jemal A, Bray F, Center MM, Ferlay J, Ward E, Forman D. Global cancer statistics. CA Cancer J Clin. 2011;61:69-90.

2. Jung KW, Won YJ, Kong HJ, Oh CM, Lee DH, Lee JS. Cancer statistics in Korea: incidence, mortality, survival, and prevalence in 2011. Cancer Res Treat. 2014;46:109-23.

3. Frumovitz M, Sun CC, Schover LR, Munsell MF, Jhingran A, Wharton JT, et al. Quality of life and sexual functioning in cervical cancer survivors. J Clin Oncol. 2005;23:7428-36.

4. Schover LR, Fife M, Gershenson DM. Sexual dysfunction and treatment for early stage cervical cancer. Cancer. 1989;63: 204-12.

5. Barter JF, Soong SJ, Shingleton HM, Hatch KD, Orr JW Jr. Complications of combined radical hysterectomy-postoperative radiation therapy in women with early stage cervical cancer. Gynecol Oncol. 1989;32:292-6.

6. Fiorica JV, Roberts WS, Greenberg H, Hoffman MS, LaPolla JP, Cavanagh D. Morbidity and survival patterns in patients after radical hysterectomy and postoperative adjuvant pelvic radiotherapy. Gynecol Oncol. 1990;36:343-7.

7. Landoni F, Maneo A, Colombo A, Placa F, Milani R, Perego P, et al. Randomised study of radical surgery versus radiotherapy for stage Ib-IIa cervical cancer. Lancet. 1997;350:535-40.

8. James RM, Cruickshank ME, Siddiqui N; Guideline Development Group. Management of cervical cancer: summary of SIGN guidelines. BMJ. 2008;336:41-3.

9. Park JY, Kim DY, Kim JH, Kim YM, Kim YT, Kim YS, et al. Comparison of outcomes between radical hysterectomy followed by tailored adjuvant therapy versus primary chemoradiation therapy in IB2 and IIA2 cervical cancer. J Gynecol Oncol. 2012;23:226-34.

10. Zivanovic O, Alektiar KM, Sonoda Y, Zhou Q, Iasonos A, Tew WP, et al. Treatment patterns of FIGO Stage IB2 cervical can- cer: a single-institution experience of radical hysterectomy with individualized postoperative therapy and definitive radiation therapy. Gynecol Oncol. 2008;111:265-70.

11. Amendola MA, Hricak H, Mitchell DG, Snyder B, Chi DS, Long $\mathrm{HJ} 3 \mathrm{rd}$, et al. Utilization of diagnostic studies in the pretreatment evaluation of invasive cervical cancer in the United States: results of intergroup protocol ACRIN 6651/GOG 183. J Clin Oncol. 2005;23:7454-9.

12. Goyal BK, Singh H, Kapur K, Duggal BS, Jacob MJ. Value of PET-CT in avoiding multimodality therapy in operable cervical cancer. Int J Gynecol Cancer. 2010;20:1041-5.

13. Pandharipande PV, Choy G, del Carmen MG, Gazelle GS, Russell AH, Lee SI. MRI and PET/CT for triaging stage IB clinically operable cervical cancer to appropriate therapy: decision analysis to assess patient outcomes. AJR Am J Roentgenol. 2009;192:802-14.

14. Lee JY, Youm J, Kim TH, Cho JY, Kim MA, Suh DH, et al. Preoperative MRI criteria for trials on less radical surgery in Stage IB1 cervical cancer. Gynecol Oncol. 2014;134:47-51.

15. Jung DC, Kim MK, Kang S, Seo SS, Cho JY, Park NH, et al. Identification of a patient group at low risk for parametrial invasion in early-stage cervical cancer. Gynecol Oncol. 2010; 119:426-30.

16. National Comprehensive Cancer Center. Cervical cancer clinical practice guidelines in oncology (v.I.2014) [Internet]. Fort Washington, PA: National Comprehensive Cancer Center; 2014 [cited 2014 Aug 4]. Available from: http://www.nccn.org.

17. Capelle L, Stevens W, Brooks S. Patterns of care for cervical cancer in Auckland, New Zealand, 2003-2007. J Med Imaging Radiat Oncol. 2011;55:82-9.

18. Ryu HS, Kang SB, Kim KT, Chang KH, Kim JW, Kim JH. Efficacy of different types of treatment in FIGO stage IB2 cervical 
cancer in Korea: results of a multicenter retrospective Korean study (KGOG-1005). Int J Gynecol Cancer. 2007;17:132-6.

19. Galic V, Herzog TJ, Lewin SN, Neugut AI, Burke WM, Lu YS, et al. Prognostic significance of adenocarcinoma histology in women with cervical cancer. Gynecol Oncol. 2012;125:287-91.

20. Lee JY, Kim YH, Kim MJ, Kim K, Chung HH, Park NH, et al. Treatment of stage IB2, IIA bulky cervical cancer: a singleinstitution experience of neoadjuvant chemotherapy followed by radical hysterectomy and primary radical hysterectomy. Arch Gynecol Obstet. 2011;284:477-82.

21. Kim WY, Chang SJ, Chang KH, Yoo SC, Chun M, Ryu HS. Treatment patterns and outcomes in bulky stage IB2 cervical cancer patients: a single institution's experience over 14 years. Gynecol Obstet Invest. 2011;71:19-23.

22. Rungruang B, Courtney-Brooks M, Beriwal S, Zorn KK, Richard SD, Olawaiye AB, et al. Surgery versus radiation therapy for stage IB2 cervical carcinoma: a population-based analysis. Int J Gynecol Cancer. 2012;22:484-9.

23. Berveling MJ, Langendijk JA, Beukema JC, Mourits MJ, Reyners AK, Pras E. Health-related quality of life and late morbidity in concurrent chemoradiation and radiotherapy alone in patients with locally advanced cervical carcinoma. J Gynecol Oncol. 2011;22:152-60.

24. Choi HJ, Ju W, Myung SK, Kim Y. Diagnostic performance of computer tomography, magnetic resonance imaging, and positron emission tomography or positron emission tomography / computer tomography for detection of metastatic lymph nodes in patients with cervical cancer: meta-analysis. Cancer Sci. 2010;101:1471-9.

25. Marnitz S, Kohler C, Affonso RJ, Schneider A, Chiantera V, Tsounoda A, et al. Validity of laparoscopic staging to avoid adjuvant chemoradiation following radical surgery in patients with early cervical cancer. Oncology. 2012;83:346-53. 